\title{
New mass determination of (15) Eunomia based on a very close encounter with (50278) 2000CZ12
}

\author{
A. Vitagliano ${ }^{1}$ and R. M. Stoss ${ }^{2,3}$ \\ 1 Dipartimento di Chimica, Università di Napoli Federico II, Complesso Universitario di Monte S. Angelo, via Cintia, \\ 80126 Napoli, Italy \\ e-mail: alvitagl@unina.it \\ 2 Observatorio Astronómico de Mallorca, Camí de l'Observatori, 07144 Costitx, Mallorca, Spain \\ 3 Astronomisches Rechen-Institut, Mönchhofstr. 12-14, 69120 Heidelberg, Germany
}

Received 5 June 2006 / Accepted 22 June 2006

ABSTRACT

\begin{abstract}
Aims. The possibility of determining the mass of large asteroids (other than Ceres, Pallas, and Vesta) from recent close encounters with smaller bodies has been investigated, and a new and more accurate determination of the mass of the asteroid (15) Eunomia is presented.

Methods. The circumstances of the close approaches between large asteroids and the ca. 129000 numbered ones were found by a numerical integration over the past 20 years. The cases for which an appreciable perturbation could be expected were investigated by fitting the mass of the perturbing body to the available observations.

Results. A very close approach took place on March 4, 2002, between Eunomia and (50278) 2000CZ12, at a nominal distance of $55200 \mathrm{~km}$. The resulting perturbation in the mean motion of the smaller body was substantially larger than for the other detected events, amounting to ca. 0.9 arcsecs/y. The orbital elements of (50278) 2000CZ12 were fitted together with the mass of Eunomia on 106 of 110 observations spanning 1950 to 2006 , resulting in a mass of $3.25 \times 10^{19} \mathrm{~kg}\left(1.64 \times 10^{-11} M_{\text {Sun }}\right)$ with a formal $1 \sigma$ relative uncertainty of $3.7 \%$. The optimized mass is $30-60 \%$ higher and the formal error $4-7$ times smaller than previous estimates.
\end{abstract}

Key words. celestial mechanics - minor planets, asteroids

\section{Introduction}

The determination of asteroid masses is an important issue both for accurately modelling the perturbations acting on other bodies and for knowing their structure and mineralogical composition. The most reliable method for determining the mass of a given minor planet is based on analysis of the perturbations on the motion of another body (test body), typically a smaller asteroid. So far, the masses of ca. 30 minor planets (apart from Ceres, Pallas, and Vesta) have been estimated by this method, most often averaging the results obtained by the study of several independent test bodies (Michalak 2001; Chernetenko \& Kochetova 2002; Kochetova 2004). However, even after averaging several independent determinations, the uncertainties associated with the mass estimates remain rather large, ranging from about $10 \%$ in the most favourable cases to more than $50 \%$ in the worst cases. This poor accuracy derives from the perturbations being normally not much larger than the errors of the optical observations used to reveal them.

For improving the accuracy, a good strategy is obviously to improve the astrometric quality of the optical observation (Chesley et al. 2005), but it can also be to identify very close approaches, whose dynamical effects can be large even over a relatively short time span and so are likely to be clear of perturbations by other bodies. During the past eight years, the numbered asteroids have increased by a factor of 15 , as have the chances of detecting potentially useful close approaches. We report here the identification of a number of encounters that could be useful for computing improved asteroid masses in the near future. Among them, an exceptionally close approach of (50278) 2000CZ12 to (15) Eunomia was found to have occurred on March 4, 2002, in the middle of an arc of available good observations ranging from october 1998 to november 2005. This allowed a new determination of the mass of Eunomia with a remarkably low formal error, which was further reduced by including in the fitting four more precovery observations and three extra ones made on demand.

\section{Methods}

The close encounters (within $0.05 \mathrm{AU}$ ) occurred during the past 20 years between 129436 numbered asteroids were detected by using the numerical-integration software SOLEX (Vitagliano 1997), which is based on a 16th-order polynomial extrapolation method of the Bulirsh-Stoer type. This software (see also http://chemistry.unina.it/ ${ }^{\sim}$ alvitagl/solex) reproduces the DE405 ephemerides of the major planets to better than 1 milliarcsec of precision $(7$ milliarcsecs in the case of Mars). The integrator can handle very close encounters through an adaptive stepsize algorithm, and its truncation and roundoff errors are totally negligible for our purposes. The precise circumstances of minimum distance were determined during the numerical integration, by using a 5th-degree Hermite interpolation inside the integration step (normally 3 days for the above integrator), whenever a condition of minimum distance was detected to have occurred within the step.

To assess the dynamical effect of each encounter, tentative mass values were adopted, based on the IRAS diameters and 
Table 1. The dynamically most effective close encounters since 1985 (excluding Ceres, Pallas, and Vesta).

\begin{tabular}{ccccccc}
\hline \hline$\# 1$ & $\# 2$ & Date & $\begin{array}{c}\text { Min. } \\
\text { distance } \\
\left(10^{9} \mathrm{~m}\right)\end{array}$ & $\begin{array}{c}\text { Deflect. } \\
\text { angle } \\
\left({ }^{\prime \prime}\right)\end{array}$ & $\begin{array}{c}\Delta n \\
\left({ }^{\prime \prime} / \mathrm{y}\right)\end{array}$ & Design. \#1 \\
\hline 15 & 50278 & $2002 / 03 / 04$ & 0.055 & $0.80^{a}$ & $0.87^{a}$ & Eunomia \\
16 & 86691 & $2005 / 11 / 08$ & 0.162 & 0.92 & 0.42 & Psyche \\
16 & 62431 & $1991 / 12 / 05$ & 0.393 & 1.82 & 0.36 & Psyche \\
9 & 36653 & $1996 / 08 / 12$ & 0.238 & 1.18 & 0.32 & Metis \\
9 & 51207 & $1995 / 07 / 23$ & 0.096 & 0.55 & 0.29 & Metis \\
10 & 57493 & $1989 / 06 / 19$ & 0.309 & 0.59 & 0.25 & Hygiea \\
324 & 121416 & $2006 / 03 / 13$ & 0.023 & 0.68 & 0.25 & Bamberga \\
15 & 14401 & $2005 / 07 / 14$ & 2.793 & $3.59^{\mathrm{a}}$ & $0.18^{\mathrm{a}}$ & Eunomia \\
704 & 87228 & $1991 / 11 / 03$ & 0.331 & 0.22 & 0.17 & Interamnia \\
10 & 87897 & $1994 / 10 / 01$ & 1.039 & 0.28 & 0.14 & Hygiea \\
\hline
\end{tabular}

${ }^{a}$ Value recomputed using the optimized mass of Eunomia.

taxonomical class of the bodies (Tedesco et al. 2002) or on the absolute magnitude $H$ as reported in the astorb database (Bowell 2006) (http: //asteroid. lowell . edu). As a criterion for selecting the most effective encounters, rather than the widelyused deflection angle (Michalak 2000, Hilton et al. 1996), we preferred to adopt (Galád 2001) the change $\Delta n$ of the mean motion of the test body, which was computed using a formula given by Edery (2001) (see also www.ai-solutions.com/ Resources/Papers/LunarGravityAssist.PDF). This led to identifying the encounter between asteroids (15) Eunomia and (50278) 2000CZ12 as the most promising candidate for an accurate mass determination.

To analyse the motion of the test body, which was done with the SOLEX package cited above, we initially used 103 observations from the public AstDys database (http://hamilton.dm.unipi.it/astdys). The dynamical model adopted included Ceres, Pallas, Vesta, and Eunomia as perturbing asteroids, but different models were also tested to assess the sensitivity of the result to the addition of perturbing effects by other bodies. The mass of Eunomia was optimized along with the orbital elements of the test body by the standard least-squares method, using equal weights for all the observations. However, different weighting schemes and/or removal of some observations were also used, in order to assess the sensitivity of the result to the individual observations. Determining the mass of the perturbing body allowed us to produce accurate precovery ephemerides, which in turn led to identifying 4 more observations, which were included in the fitting to give the final optimized value of the mass.

\section{Results and discussion}

A selection of what are likely to be the most effective encounters between 1985 and 2006 is presented in Table 1, sorted in descending order of $\Delta n$. It is worth noting that the correlation between the deflection angle and the mean-motion variation is very weak and that the efficiency of the perturbation in producing a measurable effect is much better expressed by the mean-motion variation than by the deflection angle (Galád 2001).

Two of the encounters (Psyche/86691 on November 8, 2005 and Bamberga/121416 on March 13, 2006) should become the basis for an accurate mass determination of the larger body within a few years, but the encounter of (15) Eunomia with (50278) 2000CZ12 on March 4, 2002 already represents a most favourable case. Indeed, not only did it produce the most effective perturbation among those listed in Table 1, but also
Table 2. Additional observations of (50278) 2000CZ12.

\begin{tabular}{cccc}
\hline \hline Epoch & RA & Dec & Obs. \\
\hline 19500615.27986 & 161646.93 & -211443.7 & 261 \\
19540307.51650 & 145926.76 & -180811.6 & 261 \\
19901016.45139 & 053627.41 & +232838.8 & 261 \\
19901016.50000 & 053627.88 & +232837.9 & 261 \\
20060529.14831 & 211449.538 & -144557.30 & J86 \\
20060529.15319 & 211449.668 & -144556.42 & J86 \\
20060529.15990 & 211449.839 & -144555.31 & J86 \\
\hline
\end{tabular}

a moderately large number of observations were available for an arc of 3.5 y both before (30) and after (73) the encounter. In spite of the relatively short observational arc, the fitting on 99 equally-weighted observations (4 rejected) successfully converged on a mass value of $1.68 \times 10^{-11}$ solar masses, with the remarkably low formal error of $0.10 \times 10^{-11}$ solar masses. Based on the above value of the mass of Eunomia, accurate ephemerides of the test body were produced over the time span 1950-1998, and four precovery observations (made on June 15, 1950, March 7, 1954, and October 16, 1990) were unambiguously detected on POSS-I and POSS-II red and blue plates. Three extra data points (on May 29, 2006) were also kindly obtained on request from J. L. Ortiz and F. J. Aceituno (Instituto de Astrofisica de Andalucia) with the 1.5-m telescope at Sierra Nevada Observatory in Spain. All the extra observations were reduced by standard techniques, using USNO-B1.0 as the reference star catalog, and "Astrometrica" by H. Raab (http://www.astrometrica.at) as processing software. By including these additional data (see Table 2) in the fitting, a final optimized mass of $(1.64 \pm 0.06) \times 10^{-11}$ solar masses was obtained.

Since asteroid mass determinations are often very sensitive to the dynamical model, to the weighting scheme, and to the inclusion or removal of some observations, the fitting was repeated under a variety of different assumptions, and the results are summarised in Table 3, whose inspection shows that the mass value resulting from the fitting is fairly stable and insensitive to "perturbations" of the starting data.

Further evidence of the stability of the mass determination was obtained by performing two separate fittings of the orbital elements of the test body, one using only the pre-encounter observations, the other using only the post-encounter observations, and both neglecting the mass of Eunomia. The two independently-determined orbits were propagated up to the same epoch of the close encounter (2002/3/4.720), giving $a_{\text {pre }}=$ $2.63050355 \pm 3.4 \times 10^{-8} \mathrm{AU}$ and $a_{\text {post }}=2.63050836 \pm 3.1 \times$ $10^{-7} \mathrm{AU}$ for the respective semimajor axes. The relative change of semimajor axis is thus $\Delta a / a=(1.83 \pm 0.12) \times 10^{-6}$, which according to a numerical experiment and to the formula given by Edery $(2001)$ gives a perturbing mass of $(1.63 \pm 0.10) \times$ $10^{-11} M_{\text {Sun }}$, essentially coincident with the nominal value resulting from the "global" fitting.

Another indication of the good reliability of the present determination is given by Fig. 1, which also shows the remarkable perturbing effect of the 2002 encounter, which in turn causes a right ascension drift of more than one arcminute in less than 40 years. The figure, which for clarity is limited to epochs after 1989, shows the difference in right ascension between the perturbed and unperturbed asteroid 50278, computed by a backwards integration from the present epoch to the past, assuming for Eunomia the nominal mass value resulting from the fitting (Table 3, row 1). Then, by performing the least-squares fit only 
Table 3. Optimized values of the mass of (15) Eunomia under the conditions specified in the notes.

\begin{tabular}{|c|c|}
\hline $\begin{array}{c}\text { Mass } \\
\left(10^{-11} M_{\text {Sun }}\right) \\
\text { Error }(1 \sigma)\end{array}$ & Notes \\
\hline $\begin{array}{l}1.635 \\
0.061\end{array}$ & $\begin{array}{l}106 \text { of } 110 \text { equally weighted observations } \\
\text { (adopted nominal solution, } \\
\text { rms of residuals } 0.52^{\prime \prime} \text { ) }\end{array}$ \\
\hline $\begin{array}{l}1.674 \\
0.096\end{array}$ & $\begin{array}{c}99 \text { of } 110 \text { equally weighted observations } \\
4 \text { precovery and } 3 \text { latest observations excluded, } \\
\text { rms of residuals } 0.52^{\prime \prime}\end{array}$ \\
\hline $\begin{array}{l}1.622 \\
0.066\end{array}$ & $\begin{array}{l}110 \text { of } 110 \text { equally weighted observations } \\
\text { (rms of residuals } 0.57^{\prime \prime} \text { ) }\end{array}$ \\
\hline $\begin{array}{l}1.600 \\
0.070^{a}\end{array}$ & $\begin{array}{l}106 \text { of } 110 \text { weighted obs., rms randomly } \\
\text { assigned in the range } 0.5-3.5^{\prime \prime} \text { to each } \\
\text { observatory code, average of } 10 \text { trials }\end{array}$ \\
\hline $\begin{array}{l}1.639 \\
0.061\end{array}$ & $\begin{array}{l}\text { Less closely approaching among } \\
\text { Eunomia's } 20 \text { clones }\left(d_{\min }=55167 \mathrm{~km}\right)\end{array}$ \\
\hline $\begin{array}{l}1.634 \\
0.061\end{array}$ & $\begin{array}{l}\text { Most closely approaching among } \\
\text { Eunomia's } 20 \text { clones }\left(d_{\min }=55020 \mathrm{~km}\right)\end{array}$ \\
\hline $\begin{array}{l}1.631 \\
0.061\end{array}$ & $\begin{array}{l}6 \text { most effective direct (perturbers }{ }^{b} \text { added to } \\
\text { the dynamical model, with assumed masses } \\
10 \text { times higher than current estimates }\end{array}$ \\
\hline $\begin{array}{l}1.648 \\
0.062\end{array}$ & $\begin{array}{l}7 \text { biggest asteroids }{ }^{c} \text { (indirect perturbers) added } \\
\text { to the dynamical model, with assumed masses } \\
2 \text { times higher than from IRAS estimates }\end{array}$ \\
\hline
\end{tabular}

${ }^{a}$ maximum deviation from the average of 10 trials.

${ }^{b}$ (127) Johanna, (135) Hertha, (358) Apollonia, (377) Campania, (379) Huenna, (776) Berbericia.

c (10) Hygiea, (16) Psyche, (31) Euphrosyne, (52) Europa, (87) Sylvia, (511) Davida, (704) Interamnia.

on the post-encounter observations, and propagating the orbit backwards without taking the mass of Eunomia in account, the residuals represented by the crosses are obtained, which nicely fit on the calculated right ascension difference plot. On the contrary, by propagating the orbit with the perturbation by Eunomia taken in account, the pre-encounter residuals smoothly line-up around the zero line. In view of this additional evidence of the "goodness of fit" it appears that the formal error, although still probably optimistic, should not be far from being a good approximation of the true uncertainty.

As a final remark, we note that future observations should reduce the uncertainty of the mass determination quite substantially. For each future opposition starting from July-August 2006 up to 2017, we have simulated a set of four "virtual observations" covering an arc of about one month and having an average rms of 0.6 arcsecs in both right ascension and declination. By progressively including the virtual observations in the fitting, the formal mass uncertainty decreases more or less steadily, already becoming about 3\% in August 2006 and dropping to 1\% by October 2015. Thus, a good observational program of asteroid 50278 should produce in the next few years what is likely to be the most accurate ground-based mass determination ever

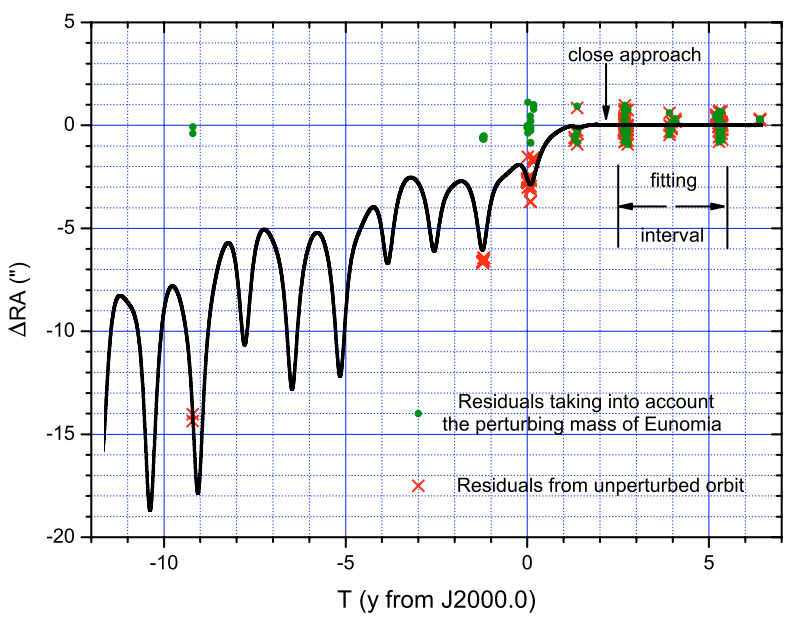

Fig. 1. Difference in the right ascension of (50278) 2000CZ12 from the perturbation by (15) Eunomia computed by backwards integration. The residuals refer to a fitting performed only on the post-encounter observations from years 2002 to 2005 . For the sake of clarity the plot is limited to epochs after 1989.

achieved for a non-binary asteroid other than Ceres, Pallas, and Vesta.

\section{Conclusions}

The present determination gives for (15) Eunomia a mass value $(1.64 \pm 0.06) \times 10^{-11} M_{\text {Sun }}$, which is $31 \%$ and $55 \%$ higher than those given by the most recent determinations: $(1.25 \pm 0.34) \times$ $10^{-11} M_{\text {Sun }}$ (Michalak 2001) and $(1.06 \pm 0.16) \times 10^{-11} M_{\text {Sun }}$ (Kochetova 2004). The formal error is substantially lower and is expected to be further reduced upon future observations of the test body (50278) 2000CZ12. This raises the estimated density of (15) Eunomia to $3.8 \pm 0.7 \mathrm{~g} / \mathrm{cm}^{3}$, based on the IRAS diameter of $255 \pm 15 \mathrm{~km}$ (Tedesco et al. 2002), which is consistent with the inferred composition (Nathues et al. 2005). In view of the improved accuracy of the mass determination, the uncertainty of the estimated density now appears to be mostly dependent on the uncertainty on the size and shape of the asteroid.

\section{References}

Bowell, E. 2006, VizieR On-line Data Catalog: B/astorb

Chernetenko, Yu., \& Kochetova, O. 2002, in Asteroids, Comets, Meteors, Berlin, 437

Chesley, S. R., Owen, W. M., Jr., Hayne, E. W., et al. 2005, American Astronomical Society, DDA meeting \#36, \#05.05

Edery, A., \& Schiff, C. 2001, 16th Intern. Symp. on Space Flight Dynamics, Pasadena

Galád, A. 2001, A\&A, 370, 311

Hilton, L., Seidelman, P. K., \& Moddour, J. 1996, AJ, 112, 2319

Kochetova, O. 2004, Sol. Syst. Res., 38, 66

Michalak, G. 2000, A\&A, 360, 363

Michalak, G. 2001, A\&A, 374, 703

Nathues, A., Mottola, S., Kaasalainen, M., \& Neukum, G. 2005, Icarus, 175, 452

Tedesco, E. F., Noah, P. V., Noah, M., \& Price, S. D. 2002, AJ, 123, 1056

Vitagliano, A. 1997, Cel. Mech. Dyn. Astr., 66, 293 